\title{
Transcriptional profile of SH-SY5Y human neuroblastoma cells transfected by Toxoplasma rhoptry protein 16
}

\author{
WEIWEI FAN ${ }^{1}$, SHUANG CHANG $^{1}$, XIUMEI SHAN ${ }^{1}$, DEJUN GAO $^{1}$, STEVEN QIAN ZHANG $^{1}$, \\ JIN ZHANG ${ }^{2}$, NAN JIANG ${ }^{2}$, DUAN MA ${ }^{2}$ and ZUOHUA MAO ${ }^{1}$ \\ ${ }^{1}$ Department of Parasitology and Microbiology; ${ }^{2}$ Key Laboratory of Molecular Medicine, Ministry of Education, \\ Department of Biochemistry and Molecular Biology, Institutes of Biomedical Sciences, School of Basic Medical Sciences, \\ Fudan University, Shanghai 200032, P.R. China
}

Received August 24, 2015; Accepted September 5, 2016

DOI: $10.3892 / \mathrm{mmr} .2016 .5758$

\begin{abstract}
Toxoplasma rhoptry protein 16 (ROP16) is crucial in the host-pathogen interaction by acting as a virulent factor during invasion. To improve understanding of the molecular function underlying the effect of ROP16 on host cells, the present study analyzed the transcriptional profile of genes in the ROP16-transfected SH-SY5Y human neuroblastoma cell line. The transcriptional profile of the SH-SY5Y human neuroblastoma cell line overexpressing ROP16 were determined by microarray analysis in order to determine the host neural cell response to the virulent factor. Functional analysis was performed using the Protein Analysis Through Evolutionary Relationships classification system. The ToppGene Suite was used to select candidate genes from the differentially expressed genes. A protein-protein interaction network was constructed using Cytoscape software according to the interaction associations determined using the Search Tool for the Retrieval of Interacting Genes/Proteins. Reverse transcription-quantitative polymerase chain reaction (RT-qPCR) analysis of the selected genes confirmed the results of the microarray. The results showed that 383 genes were differentially expressed in response to ROP16 transfection, of which 138 genes were upregulated and 245 genes were downregulated. Functional analysis indicated that the differentially expressed genes (DEGs) were involved in several biological processes, including
\end{abstract}

Correspondence to: Professor Zuohua Mao, Department of Parasitology and Microbiology, School of Basic Medical Sciences, Fudan University, 130 Dong'an Road, Shanghai 200032, P.R. China E-mail: zhmao@shmu.edu.cn

Professor Duan Ma, Key Laboratory of Molecular Medicine, Ministry of Education, Department of Biochemistry and Molecular Biology, Institutes of Biomedical Sciences, School of Basic Medical Sciences, Fudan University, 130 Dong'an Road, Shanghai 200032, P.R. China

E-mail: duanma@fudan.edu.cn

Key words: Toxoplasma gondii, rhoptry protein 16, microarray, transcriptome, bioinformatics developmental process, biological regulation and apoptotic process. A total of 15 candidate genes from the DEGs were screened using the ToppGene Suite. No significant differences in expression were observed between the RT-qPCR data and the microarray data. Transfection with ROP16 resulted in alterations of several biological processes, including nervous system development, apoptosis and transcriptional regulation. Several genes, including CXCL12, BAI1, ZIC2, RBMX, RASSF6, MAGE-A6 and HOX, were identified as significant DEGs. Taken together, these results may contribute to understanding the mechanisms underlying the functions of ROP16 and provide scope for further investigation of the pathogenesis of Toxoplasma gondii.

\section{Introduction}

Toxoplasma gondii (T. gondii) is an opportunistic protozoan pathogen, which is capable of invading and replicating in all nucleated cells. T. gondii infection can cause neurological problems, including encephalitis, in immunocomprised individuals (1). Of note, toxoplasmic infection in the prenatal period is a common cause of brain malformation, and can critically affect the central nervous system and retinal development, culminating in long-term cognitive deficits (2). Histopathological lesions in the frontal lobe have been found to be more prevalent than in other areas of the brain of BALB/c mice infected with $T$. gondii (3). $T$. gondii is defined by the presence of an apical complex, including secretory organelles $(4,5)$. The rhoptries are one type of apical secretory organelle of $T$. gondii, and they have been shown to be closely associated with the parasites' pathogenesis, host cell invasion and host cell interaction (6). At present, $>30$ rhoptry proteins have been identified, with several being involved in the invasive process, and important for growth and survival in the host cell (4). ROP16 has serine-threonine kinase activity, a molecular weight of $96 \mathrm{kD}$ and comprises 707 amino acids. The uniqueness of ROP16 to the apicomplexa is crucial in the host-pathogen interaction (7). ROP16 is a key virulence determinant in terms of being a member of the ROP2 family and being able to invade the host cell nucleus rapidly following parasite infection. This protein invades host cell and accumulates in the host cell nucleus due to the nucleus localized 
sequence (8). ROP16, a regulator of host cell transcription, subverts the host functions by direct tyrosine phosphorylation of the signal transducer and activator of transcription (STAT) pathways. Evidence obtained has confirmed that ROP16 from type I or III strains of $T$. gondii can affect the activation of the STAT3/6 signaling pathways, with consequent downstream effects on the key host cytokine, interleukin-12 (9). In addition, ROP16 induces the phosphorylation and nuclear translocation of STAT5 to generate protective immunity (10). These findings show that ROP16 can affect and subvert gene functions in the nucleus. The present study investigated the gene expression profile in the SH-SY5Y human neuroblastoma cell line transfected with ROP16 in order to improve understanding of the molecular function of ROP16 in the host neurocyte.

\section{Materials and methods}

Cell culture. The SH-SY5Y cells (American Type Culture Collection, Manassas, VA, USA) were cultured in Dulbecco's modified Eagle's medium (DMEM; Hyclone; GE Healthcare Life Sciences, Logan UT, USA) supplemented with $10 \%$ heat-inactivated fetal bovine serum (FBS; Gibco; Thermo Fisher Scientific, Inc., Waltham, MA, USA). The cells were incubated at $37^{\circ} \mathrm{C}$ in a humidified air atmosphere containing $5 \% \mathrm{CO}_{2}$ and were passaged every 2-4 days by trypsinization.

Transfection of cells with ROP16. The T. gondii ROP16 gene (preserved in house) was amplified via polymerase chain reaction (PCR) analysis using the following primers: ROP16, forward 5'-GAGAATTCCATGAAAGTGACCACGAAA GG-3', containing EcoRI, and reverse 5'-GCGATATCCTTG TCATCGTCGTCCTTGTAGTCCATCCGATGTGAAGAA AGTTC-3', containing the Flag-tag gene sequence EcoRV, and ligated using the NEB kit (New England Biolabs, Inc., Ipswich, MA, USA) with the target vector, pPEXPR-IBA105IP (preserved in house). The cycling conditions for PCR were as follows: i) Initial denaturation at $94^{\circ} \mathrm{C}$ for $5 \mathrm{~min}$, ii) denaturation at $94^{\circ} \mathrm{C}$ for $30 \mathrm{sec}$, iii) annealing at $65^{\circ} \mathrm{C}$ for $30 \mathrm{sec}$, iv) elongation at $72^{\circ} \mathrm{C}$ for $150 \mathrm{sec}$ (repeat steps ii-iv for 30 cycles), vi) final extension at $72^{\circ} \mathrm{C}$ for $10 \mathrm{~min}$. The vector DNA and Lipofectamine 2000 (Invitrogen; Thermo Fisher Scientific, Inc.) were mixed together following incubation at room temperature for $5 \mathrm{~min}$. The mixture was added to 6 -well culture plates (density, $~ 80 \%$ ) following incubation at room temperature for $20 \mathrm{~min}$. The mixture was then incubated in $5 \% \mathrm{CO}_{2}$ at $37^{\circ} \mathrm{C}$. Puromycin $(1 \mu \mathrm{g} / \mathrm{ml})$ was used to select stably transfected cells following 24-48 h of culture. The SH-SY5Y-ROP16 cell line was cultured in the same conditions as the SH-SY5Y cell line. Finally, the expression level of ROP16 in the SH-SY5Y was determined using western blot analysis.

RNA isolation and microarray analysis. The total RNA of each cell line was extracted using TRIzol reagent (Invitrogen; Thermo Fisher Scientific, Inc.) according to the manufacturer's protocol. The RNA was further purified using a NucleoSpin ${ }^{\circledR}$ RNA clean-up kit (Macherey-Nagel, Düren, Germany) and was quantified using an ultraviolet spectrophotometer and formaldehyde denaturing agarose gel electrophoresis. The RNA samples were sent to the Bioassay
Laboratory of CapitalBio Corporation (Beijing, China) for microarray hybridization and analysis. Briefly, total RNA from each sample was reverse transcribed by denaturation at $94^{\circ} \mathrm{C}$ for $5 \mathrm{~min}, 94^{\circ} \mathrm{C}$ for $30 \mathrm{sec}$, annealing at $55^{\circ} \mathrm{C}$ for $30 \mathrm{sec}$ and elongation at $68^{\circ} \mathrm{C}$ for $150 \mathrm{sec}$, with a final extension at $68^{\circ} \mathrm{C}$ for $5 \mathrm{~min}$. The cDNA synthesized was then labeled with CY3-dCTP (cat. no. PA 53021; GE Healthcare Life Sciences) using klenow enzyme (New England Biolabs, Inc.) according to the manufacturer's protocol. The hybridized slides were scanned on an Agilent G2565CA microarray scanner (Agilent Technologies, Inc., Santa Clara, CA, USA). The resulting text files were then obtained from the scanned image using Agilent Feature Extraction image analysis software and imported to Agilent GeneSpring GX software (Agilent Technologies, Inc.). The microarray data was normalized using the percentile shift method.

Analysis of differentially expressed genes (DEGs). In the present study, a 2 -fold change ( $\log 2$ fold-change $\geq 1$ or $\leq-1$ ) and a flag tag of 'Detected' were used as the thresholds to identify the DEGs. A gene was considered to be differentially expressed by transfection of ROP16 if its expression increased or decreased by $\geq 2$-fold, compared with the control sample. Gene Ontology(GO) analysis was performed using Protein Analysis Through Evolutionary Relationships (PANTHER) for biological processes $(11,12)$. The ToppGene Suite was used in order to select candidate genes of $T$. gondii from the microarray data (13). DEGs from the microarray data were defined as the 'test gene set' and genes associated with $T$. gondii were defined as the 'training gene set' according to the records of Online Mendelian Inheritance in Man (OMIM; http://omim.org/) and GeneCards (http://www.genecards.org/) databases. The Search Tool for the Retrieval of Interacting Genes/Proteins (STRING; http://www.string-db.org/) online database was used to evaluate information on protein-protein interactions (PPIs) of the DEGs (14). Interactions with a high required confidence with a combined score $\geq 0.7$ were selected. According to these interactions, PPI networks were constructed using Cytoscape software (3.2.1; http://cytoscape.org/) (15). Further module analysis of the networks was performed using Molecular Complex Detection (MCODE), which is an application in Cytoscape (16). The parameters used to identify the enriched functional modules were as follows: Degree cutoff, 2; Node score cutoff, 0.2; K-Core, 2; Maximum depth, 100.

$R T$-qPCR analysis. RT-qPCR analysis was used to confirm the microarray results in the present study. The cDNA was synthesized in a $20 \mu \mathrm{l}$ reaction system. Genomic DNA was extracted using 2.0 $\mu \mathrm{l}$ 5xgDNA Eraser Buffer, $1.0 \mu \mathrm{l}$ gDNA Eraser, $1 \mu \mathrm{g}$ total RNA and complemented RNase-free $\mathrm{dH}_{2} \mathrm{O}$ up to $10 \mu \mathrm{l}$. The above mixture was incubated at $42^{\circ} \mathrm{C}$ for 2 min. Subsequently, $4.0 \mu 15 \mathrm{X}$ PrimeScript ${ }^{\circledR}$ Buffer 2 (for Real Time), $1.0 \mu$ l PrimeScript ${ }^{\circledR}$ RT Enzyme mix I, $1.0 \mu \mathrm{l}$ RT Primer mix and complemented RNase-free $\mathrm{H}_{2} \mathrm{O}$ up to $20 \mu \mathrm{l}$ were added. The mixture was incubated at $37^{\circ} \mathrm{C}$ for $15 \mathrm{~min}$ and $85^{\circ} \mathrm{C}$ for $5 \mathrm{sec}$ to generate cDNA. The prepared cDNA was stored at $-20^{\circ} \mathrm{C}$. SYBR Green Real-time PCR Master mix (Takara Bio, Inc., Otsu, Japan) was used to perform the reaction, a $10 \mu \mathrm{l}$ volume contained $2.5 \mu \mathrm{l} \mathrm{SYBR}{ }^{\circledR}$ Premix Ex TaqTM II (2X), $0.2 \mu \mathrm{l}$ PCR forward primer 
Table I. Primers used in Reverse transcription-quantitative polymerase chain reaction analysis.

\begin{tabular}{lll}
\hline Gene symbol & \multicolumn{1}{c}{ Forward primer (5'-3') } & \multicolumn{1}{c}{ Reverse primer (5'-3') } \\
\hline ABCA1 & ACCCACCCTATGAACAACATGA & GAGTCGGGTAACGGAAACAGG \\
STAT4 & GCTTAACAGCCTCGATTTCAAGA & GAGCATGGTGTTCATTAACAGGT \\
BMP2 & ACTACCAGAAACGAGTGGGAA & GCATCTGTTCTCGGAAAACCT \\
S1PR5 & GCGCACCTGTCCTGTACTC & SGTTGGTGAGCGTGTAGATGATG \\
TPM2 & AGTTTGCCGAGGGTCTGTG & TGGTCCAAGGTCTGGTGAATC \\
ISL1 & GCGGAGTGTAATCAGTATTTGGA & GCATTTGATCCCGTACAACCT \\
SDK2 & TCAAGTGGCTCCACAACAACA & GCACGATGCAACGGTAAAAGC \\
SEMA3D & TCAGAGCACTACTGGCTCAAT & ATCGGAGGTACTGCCTTCTTG \\
SP2 & CTCAGCCCCGGCAAGAATAG & TTGATCGGGTCCCTTTGTTGA \\
ACSBG1 & AGTACATCGCTTATGACTGCTGC & TGGGTGTCAATGATGGCGTC \\
GAPDH & ACAACTTTGGTATCGTGGAAGG & GCCATCACGCCACAGTTTC
\end{tabular}

(10 $\mu \mathrm{M}), 0.2 \mu \mathrm{l}$ PCR reverse primer $(10 \mu \mathrm{M}), 0.1 \mu \mathrm{l} \mathrm{ROX}$

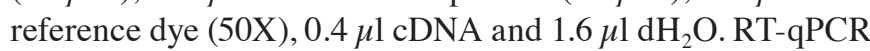
analysis was performed on a StepOnePlus Real-Time PCR system (Applied Biosystems; Thermo Fisher Scientific, Inc.) according to the manufacturer's protocol. The housekeeping gene, glycerldehyde-3-phosphate dehydrogenase (GAPDH) was selected as an internal reference, and 10 additional genes were selected at random from the DEGs, the primers of which are shown in Table I. Triplicate RT-qPCR amplifications were performed for each gene. The amplification parameters were set as follows: $95^{\circ} \mathrm{C}$ for $30 \mathrm{sec}, 95^{\circ} \mathrm{C}$ for $5 \mathrm{sec}, 60^{\circ} \mathrm{C}$ for $31 \mathrm{sec}$ over 40 cycles. Finally, the quantification values were calculated and analyzed using the $2^{-\Delta \Delta C q}$ method (17).

\section{Results}

Transfection of cells with ROP16. The present study used GAPDH as an internal reference for western blot analysis in order to compare the transcriptional levels of ROP16 in the SH-SY5Y-ROP16 and SH-SY5Y cell lines. The result demonstrated that ROP16 was successfully transfected into the SH-SY5Y cells (Fig. 1).

Response of DEGs to transfection with ROP16. The microarray data revealed that the host cell genes exhibited significant variety in expression level due to the transfection. A total of 383 genes were significantly altered as DEGs, which included 138 upregulated genes and 245 downregulated genes (Fig. 2).

Functional categories of DEGs. The DEGs were analyzed using PANTHER in accordance with the biological process of PANTHER GO slim (a subset of GO terms providing a broad overview of GO) to elucidate the correlation between genes and physiology in host cells. As revealed by the results, transfection of cells with ROP16 affected several biological processes, including apoptotic process, biological regulation, development process, metabolic process and response to stimulus (Fig. 3). The details of the biological processes of these genes are listed in Table II. Genes associated with neural system development, apoptosis and transcriptional regulation are listed in Table III according to the functional categories.

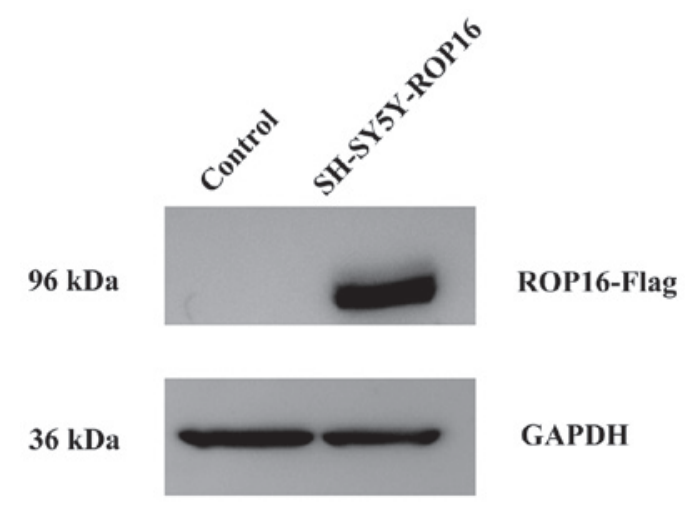

Figure 1. Western blot detection of ROP16 in SH-SY5Y-ROP16 and SH-SY5Y cells. ROP16 rhoptry protein 16; GAPDH, glycerldehyde-3-phosphate dehydrogenase.

Candidate genes derived from DEGs. According to the instructions of ToppGene and ToppNet, the 'training gene set' contained 12 genes associated with Toxoplasma, which were collected from the OMIM and GeneCards database searches (Fig. 4). Prioritization of candidate genes was obtained using the ToppGene method and ToppNet method. Candidate genes selected from the top 30 of the ToppGene and ToppNet ranks are shown in Table IV. In addition, 15 genes (CXCL12, APOE, STAT4, ABCA1, CISH, BMP2, ATG16L1, IGF8, PMS2, ATXN2, ELK1, PSMD9, AMOT, IARS and ISL1) were present in both sets of results.

PPI network construction. A total of 40 PPI associations were obtained using STRING, and networks were constructed using Cytoscape (Fig. 5). Typically, an individual network comprised a small gene count. S1PR5, CXCL12, LPAR3 and SSTR4 showed a high degree of connectivity, which was the only cluster examined by MCODE. The network was selected as a significant module by MCODE, with an MCODE score of four.

Validation of microarray results. To confirm the microarray results, RT-qPCR analysis was performed on 10 selected genes (ABCA1, STAT4, BMP2, S1PR5, TPM2, ISL1, SDK2, 
Table II. Biological processes categories of differentially expressed genes.

\begin{tabular}{|c|c|c|c|}
\hline Biological process & Count & $\begin{array}{l}\text { Percentage gene } \\
\text { hits, vs. total }\end{array}$ & $\begin{array}{c}\text { Process hits } \\
(\%)\end{array}$ \\
\hline \multicolumn{4}{|l|}{ Upregulated differentially expressed genes } \\
\hline Cellular component organization or biogenesis (GO:0071840) & 7 & 0.06 & 0.04 \\
\hline Cellular process (GO:0009987) & 28 & 0.24 & 0.17 \\
\hline Localization (GO:0051179) & 16 & 0.14 & 0.10 \\
\hline Apoptotic process (GO:0006915) & 3 & 0.02 & 0.02 \\
\hline Reproduction (GO:0000003) & 5 & 0.04 & 0.03 \\
\hline Biological regulation (GO:0065007) & 23 & 0.20 & 0.14 \\
\hline Response to stimulus (GO:0050896) & 3 & 0.03 & 0.02 \\
\hline Developmental process (GO:0032502) & 17 & 0.15 & 0.10 \\
\hline Multicellular organismal process (GO:0032501) & 11 & 0.10 & 0.07 \\
\hline Biological adhesion (GO:0022610) & 1 & 0.01 & 0.01 \\
\hline Metabolic process (GO:0008152) & 49 & 0.42 & 0.29 \\
\hline Immune system process (GO:0002376) & 6 & 0.06 & 0.04 \\
\hline \multicolumn{4}{|l|}{ Downregulated differentially expressed genes } \\
\hline Cellular component organization or biogenesis (GO:0071840) & 11 & 0.05 & 0.03 \\
\hline Cellular process (GO:0009987) & 66 & 0.30 & 0.18 \\
\hline Localization (GO:0051179) & 26 & 0.12 & 0.07 \\
\hline Apoptotic process (GO:0006915) & 6 & 0.03 & 0.02 \\
\hline Reproduction (GO:0000003) & 14 & 0.06 & 0.04 \\
\hline Biological regulation (GO:0065007) & 40 & 0.18 & 0.11 \\
\hline Response to stimulus (GO:0050896) & 14 & 0.06 & 0.04 \\
\hline Developmental process (GO:0032502) & 37 & 0.17 & 0.10 \\
\hline Multicellular organismal process (GO:0032501) & 33 & 0.15 & 0.09 \\
\hline Biological adhesion (GO:0022610) & 12 & 0.06 & 0.03 \\
\hline Metabolic process (GO:0008152) & 92 & 0.42 & 0.25 \\
\hline Immune system process (GO:0002376) & 14 & 0.06 & 0.04 \\
\hline
\end{tabular}

GO, Gene Ontology.

SEMA3D, SP2 and ACSBG1). These genes were all in accordance with the microarray data, although individual values were not identical due to differences in sensitivity between the two methods (Fig. 6).

\section{Discussion}

Identifying novel candidate genes of certain diseases provides clues for the investigation of novel biomarkers or target genes for diagnosis or therapy, and improves understanding of pathogenesis. ToppGene Suite is an advanced bioinformatics tool for examining and prioritizing candidate genes through comprehensive assessment of factors, including GO annotation, phenotype, signaling pathway and protein interaction, from a specific gene list $(13,18)$. In the present study, 383 genes were found to have significantly altered expression through microarray-based detection comparing the expressional profiles of SH-SY5Y-ROP16 with those of SH-SY5Y. A total of 138 genes were upregulated and 245 genes were downregulated due to transfection with ROP16. The present study attempted to screen the most likely candidate gene associated with Toxoplasma in the DEGs following transfection with ROP16. The results of ToppGene and ToppNet revealed which DEGs caused by transfection with ROP16 were the most likely candidate genes associated with Toxoplasma and may be further investigated as target genes. The intersecting genes from ToppGene and ToppNet included CXCL12, APOE, STAT4, ABCA1, CISH, BMP2, ATG16L1, IGF8, PMS2, ATXN2, ELK1, PSMD9, AMOT, IARS and ISL1. Intersection indicated that the gene warranted high prioritization. The data in the present study showed that CXCL12 had the highest prioritization with a downregulated expression level, which may indicate that CXCL12 was a critical factor associated with the state of host cell development in response to the ROP16 transfection. CXCL12 is a key innate immune mediator, which is involved in embryonic neuronal survival and migration. CXCL12 is generally expressed in the brain of the central nervous system. A previous study noted that CXCL12 maintains the development of motor neurons and axons (19), and also contributes to the development of glioma (20). CXCL12 was involved in a significant network module with S1PR5, SSTR4 and LPAR3 according to the PPI network analysis. This suggested that these four genes may be considered as potential targets for the interaction between Toxoplasma and host. 


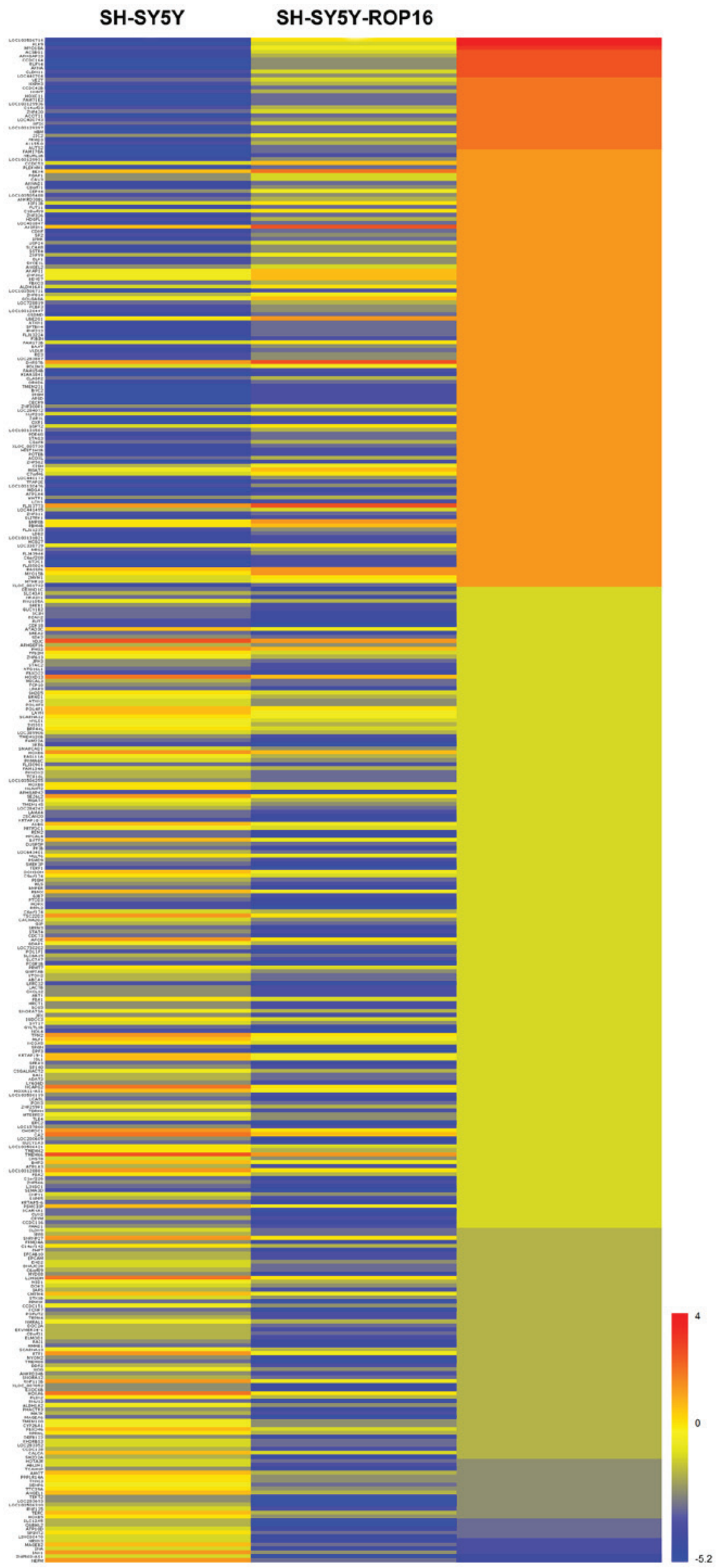

Figure 2. Transcriptional response of SH-SY5Y transfected with ROP16. Change from red to blue indicates movement from upregulation to downregulation. ROP16 rhoptry protein 16. 
Table III. Genes associated with nervous system development, apoptosis and transcriptional regulation.

Functional category of interest
Gene symbol

Upregulated genes $\quad$ Downregulated genes

Nervous system development

HOXC11, NEURL1B, BNC2, ZIC2, RBM4B, SLITRK1, PCBP3

CISH, RASSF6

HOXC11, USP24, ZIC2, TFAP2E, PDLIM3, WWTR1, ZMYM1, ZNF582, ANGEL2, ZNF814, POTEB, ZNF99, LDB2, NFIX, GSPT2, MRS2, RBM4B, ELK1, SLITRK1, HDGFL1, SP2, PCBP3
HOXB6, SDK2, HOXB5, IGDCC3, SH2D5, HOXD13, SEMA3D, SCG5, HOXB8, TPM2, RASL11A, ATXN2, LINGO1, BAI1, SYT17, RBMX, CDK18, ABLIM1, PHACTR3, EHD2, ISL1, SLC6A15, REM2, NRXN3, HOXA6, DOC2A, LAMA4

MAGEA6, SDK2, IGDCC3, MAGEB2, STAT4, DPF3

ZNF566, GNPTAB, BATF3, HOXB6, POU4F3, HOXB5, CUX2, ZSCAN20, SMARCAD1, HOXD13, ZNF613, KHDRBS3, TLE4, HOXB8, POU4F1, RAI1, TSC22D3, PKNOX2, TFB2M, PMS2, LINGO1, RBMX, IRF8, IARS, ISL1, POU1F1, GDAP1, STAT4, PIGM, MLLT6, ANGEL1, HOXA6, SP140, DPF3

\section{GO Biological Process of Up-regulated Genes}

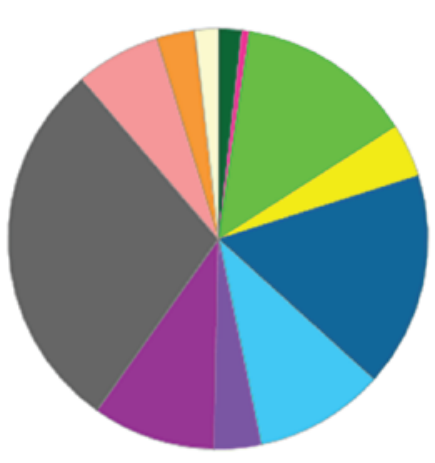

(GO:0006915) apoptotic process (GO:0022610) biological adhesion (GO:0065007) biological regulation (GO:0071840) cellular component organization or biogenesis (GO:0009987) cellular process (GO:0032502) development process (GO:0002376) immune system process (GO:0051179) localization (GO:0008152) metabolic process (GO:0032501) multicellular organismal process (GO:0000003) reproduction (GO:0050896) response to stimulus

\section{GO Biological Process of Down-regulated Genes}

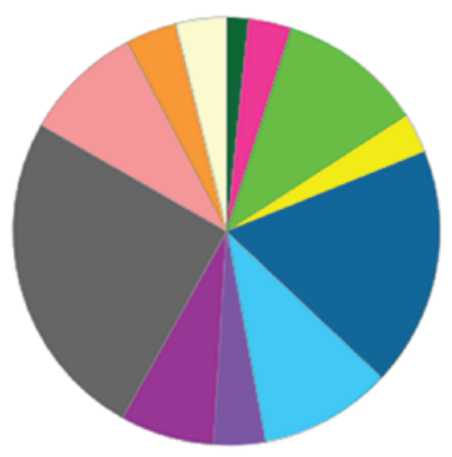

(GO:0006915) apoptotic process (GO:0022610) biological adhesion (GO:0065007) biological regulation (GO:0071840) cellular component organization or biogenesis (GO:0009987) cellular process (GO:0032502) development process (GO:0002376) immune system process (GO:0051179) localization (GO:0008152) metabolic process (GO:0032501) multicellular organismal process (GO:0000003) reproduction (GO:0050896) response to stimulus 
Table IV. Top 30 candidate genes of ToppGene and ToppNet prioritization results.

\begin{tabular}{|c|c|c|c|c|c|c|c|}
\hline \multicolumn{4}{|c|}{ ToppGene } & \multicolumn{4}{|c|}{ ToppNet } \\
\hline Rank & $\begin{array}{c}\text { Gene } \\
\text { symbol }\end{array}$ & $\begin{array}{c}\text { Average } \\
\text { score }\end{array}$ & $\begin{array}{l}\text { Overall } \\
\text { P-value }\end{array}$ & Rank & $\begin{array}{c}\text { Gene } \\
\text { symbol }\end{array}$ & $\begin{array}{c}\text { Interactant } \\
\text { count }\end{array}$ & Score \\
\hline 1 & CXCL12 & 0.95112 & 4.83E-09 & 1 & STAT4 & 14 & $2.34 \mathrm{E}-03$ \\
\hline 2 & APOE & 0.72009 & $5.09 \mathrm{E}-07$ & 2 & TERF1 & 299 & 7.37E-04 \\
\hline 3 & STAT4 & 0.78586 & $1.08 \mathrm{E}-05$ & 3 & ATXN1 & 255 & 4.48E-04 \\
\hline 4 & ABCA1 & 0.73670 & $1.91 \mathrm{E}-05$ & 4 & CXCL12 & 10 & $3.94 \mathrm{E}-04$ \\
\hline 5 & CISH & 0.70228 & 2.13E-04 & 5 & CISH & 27 & $3.82 \mathrm{E}-04$ \\
\hline 6 & SSTR4 & 0.72088 & 4.10E-04 & 6 & SRGN & 11 & $2.47 \mathrm{E}-04$ \\
\hline 7 & BMP2 & 0.67482 & $4.48 \mathrm{E}-04$ & 7 & RBMX & 89 & $1.61 \mathrm{E}-04$ \\
\hline 8 & ATG16L1 & 0.64074 & 4.59E-04 & 8 & APOE & 54 & $1.56 \mathrm{E}-04$ \\
\hline 9 & CALCA & 0.57179 & 4.87E-04 & 9 & SMARCAD1 & 119 & $1.43 \mathrm{E}-04$ \\
\hline 10 & IRF8 & 0.54026 & $7.56 \mathrm{E}-04$ & 10 & HOXC11 & 13 & $1.27 \mathrm{E}-04$ \\
\hline 11 & PMS2 & 0.58553 & 7.61E-04 & 11 & ABCA1 & 48 & $1.05 \mathrm{E}-04$ \\
\hline 12 & LAMA4 & 0.53700 & $9.43 \mathrm{E}-04$ & 12 & AMOT & 69 & $9.70 \mathrm{E}-05$ \\
\hline 13 & BATF3 & 0.65302 & 0.001017 & 13 & HIST1H1B & 42 & $9.64 \mathrm{E}-05$ \\
\hline 14 & ATXN2 & 0.54901 & 0.001293 & 14 & TPM2 & 35 & $9.59 \mathrm{E}-05$ \\
\hline 15 & ELK1 & 0.45828 & 0.001894 & 15 & ELK1 & 41 & $9.49 \mathrm{E}-05$ \\
\hline 16 & WLS & 0.61716 & 0.002097 & 16 & IARS & 69 & $9.14 \mathrm{E}-05$ \\
\hline 17 & MGAT2 & 0.38112 & 0.002123 & 17 & PTCD3 & 32 & $8.18 \mathrm{E}-05$ \\
\hline 18 & MYO18A & 0.47104 & 0.003082 & 18 & CDC73 & 61 & $8.06 \mathrm{E}-05$ \\
\hline 19 & PSMD9 & 0.43535 & 0.003175 & 19 & PSMD9 & 38 & $7.48 \mathrm{E}-05$ \\
\hline 20 & WWTR1 & 0.53933 & 0.003483 & 20 & SH2D2A & 12 & $6.98 \mathrm{E}-05$ \\
\hline 21 & AMOT & 0.49193 & 0.003639 & 21 & ATG16L1 & 35 & $6.76 \mathrm{E}-05$ \\
\hline 22 & LPAR3 & 0.58023 & 0.003655 & 22 & BMP2 & 19 & $6.06 \mathrm{E}-05$ \\
\hline 23 & IARS & 0.47188 & 0.003673 & 23 & IRF8 & 22 & $6.05 \mathrm{E}-05$ \\
\hline 24 & GFRA3 & 0.69785 & 0.003804 & 24 & RTF1 & 26 & $5.94 \mathrm{E}-05$ \\
\hline 25 & DDR2 & 0.54028 & 0.003883 & 25 & KHDRBS3 & 29 & $5.75 \mathrm{E}-05$ \\
\hline 26 & ISL1 & 0.64497 & 0.003949 & 26 & ATXN2 & 38 & $5.75 \mathrm{E}-05$ \\
\hline 27 & S1PR5 & 0.60005 & 0.004607 & 27 & ISL1 & 20 & $5.30 \mathrm{E}-05$ \\
\hline 28 & EPCAM & 0.51005 & 0.004612 & 28 & MID1 & 30 & $5.27 \mathrm{E}-05$ \\
\hline 29 & VLDLR & 0.53509 & 0.004849 & 29 & PMS2 & 44 & $5.20 \mathrm{E}-05$ \\
\hline 30 & FBXO22 & 0.45987 & 0.005457 & 30 & PDE6G & 8 & $5.04 \mathrm{E}-05$ \\
\hline
\end{tabular}

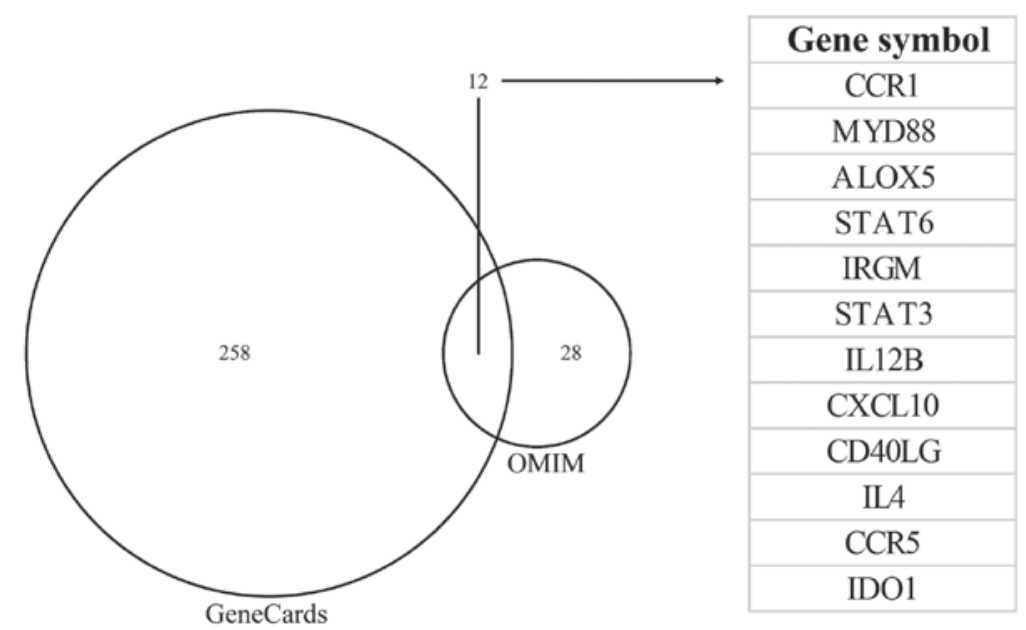

Figure 4. Training gene set for ToppGene derived from OMIM and GeneCards databases. Searches for the key phrases, 'Toxoplasma gondii' OR 'Toxoplasmosis' OR 'Toxoplasma' OR 'T. gondii' in the two databases, led to the detection of 40 genes from OMIM and 270 genes from GeneCards. The 12 genes from the intersection of the two databases were defined as the 'training gene set'. OMIM, Online Mendelian Inheritance in Man. 

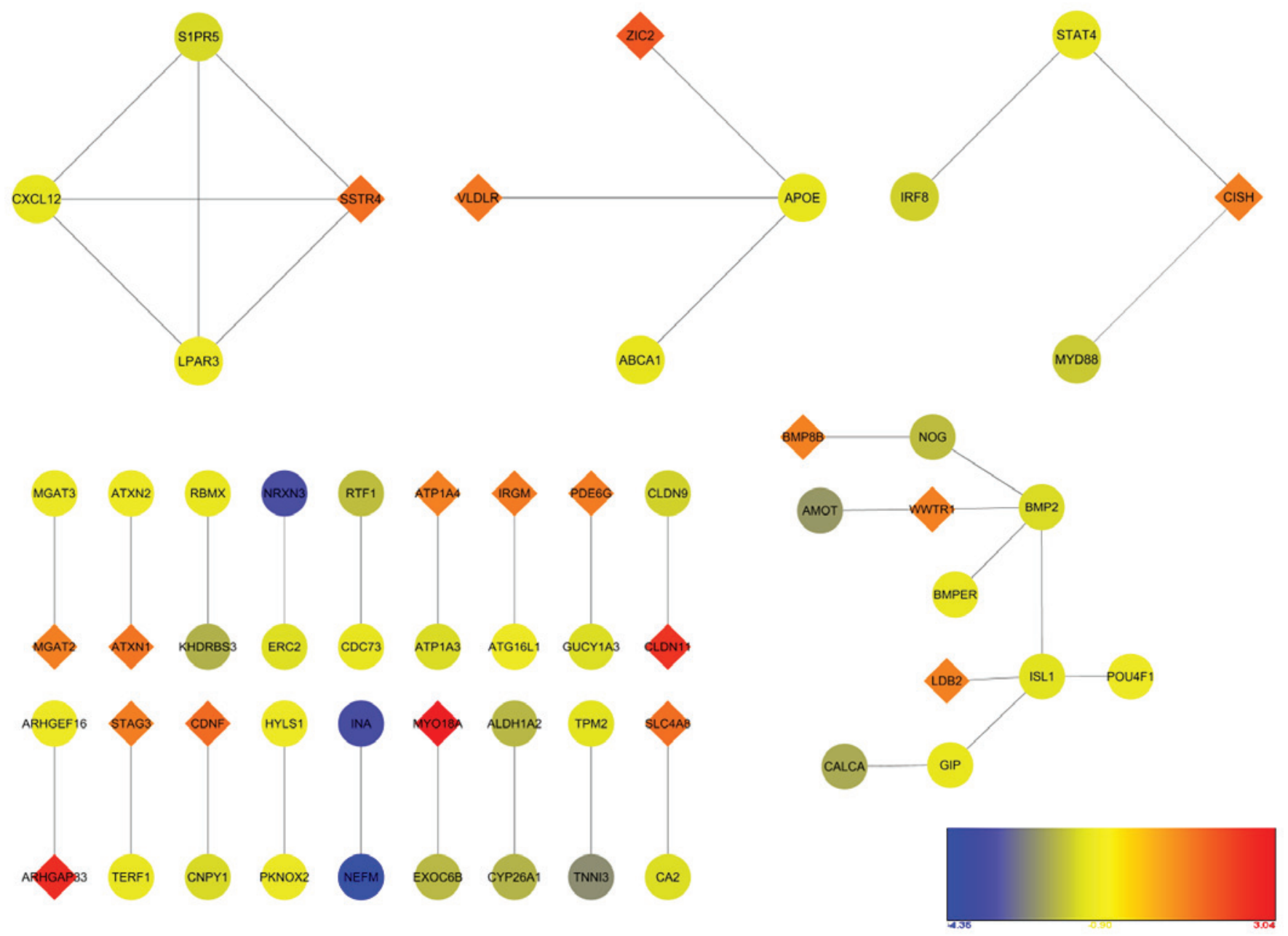

Figure 5. Protein-protein interaction network of DEGs. Nodes represent proteins, edges represent interactions between proteins, diamonds represent upregulated DEGs and circles represent downregulated DEGs. The color of the node, with a gradual change between blue and red, correspond to the variation between downeregulation and upregulation. of the expression of the DEG. DEG, differentially expressed gene.

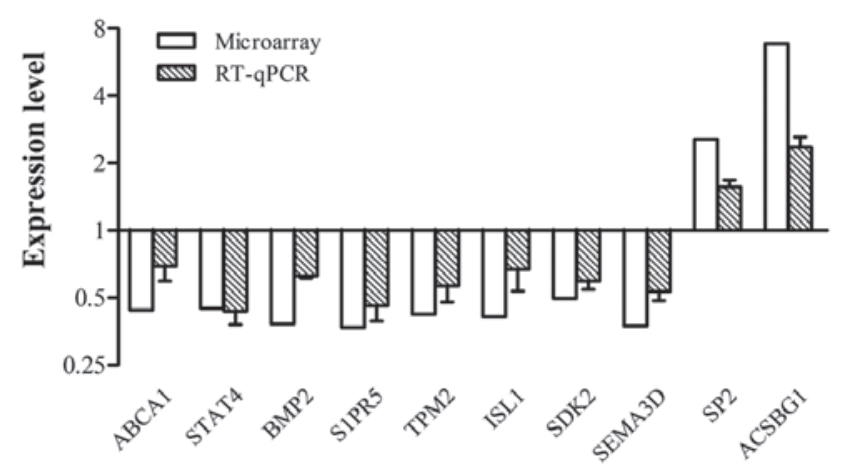

Genes

Figure 6. Validation of the microarray results by comparative RT-qPCR analysis. Data are presented as the mean \pm standard deviation. RT-qPCR, reverse transcription-quantitative polymerase chain reaction.

All DEGs analyzed by PANTHER in the present study were involved in several biological processes, including cellular component organization or biogenesis, cellular process, localization, apoptotic process, reproduction, biological regulation, response to stimulus, developmental process, multicellular organismal process, biological adhesion, metabolic process and immune system process. The human nervous system is vital in maintaining homeostasis, comprising the central nervous system and peripheral nervous system. The brain is a preferred target during infection, particularly for chronic $T$. gondii infections. T. gondii may interfere with host neuronal function and modulate signaling pathways in the host brain (21). The expressional profiles revealed 23 genes (six upregulated and 17 downregulated) associated with nervous system development, a subclass of developmental process. The expression of BAI1 was downregulated by the transfection with ROP16 among these genes. BAI1 is known as an angiogenesis inhibitor, which can be directly induced by wild-type p53 and has been reported to be specifically expressed in the human brain. BAI1 is typically expressed in the cerebral cortex of the human adult brain, and regulates synaptic plasticity and signal transduction in the neurological system $(22,23)$. The ZIC2 gene is upregulated as it is a vital gene in human brain, which is involved in neural development (24). RBMX is a protein-coding gene for the production of an RNA-binding protein involved in the tissue-specific regulation of gene transcription and alternative splicing of several pre-mRNAs. A previous study showed that a low expression level of RBMX resulted in poor development of the zebrafish head (25). The downregulated expression of RBMX was also shown in the 
present study, which may indicate that the central nervous system was disturbed in the SH-SY5Y cells due to transfection with ROP16. Taken together, the decreased expression levels of BAI1 and RBMX, and increased expression of ZIC2 following transfection with ROP16 disrupted nervous system development.

Apoptosis or 'programmed' cell death is an essential process in cells. Apoptosis is involved in healthy cells and diseased cells. It occurs during the normal development of cells and maintains the population of cells in tissues. Apoptosis acts as a defense mechanism, which removes toxins and contributes to homeostasis when immune reactions are activated. Previous studies have revealed that $T$. gondii infection results in cell apoptosis through disturbing signaling pathways of host cells $(26,27)$. In the present study, nine genes associated with apoptosis were detected using microarray analysis, including three upregulated genes and six downregulated genes. It has been found that the upregulated gene, RASSF6, can interact with MDM2 and stabilize p53, promoting apoptosis and G1/S arrest in HCT116 cell lines (28). The present study showed that RASSF6 was upregulated, which indicate that similar pathways may be involved in the apoptotic process of SH-SY5Y cells. It is well known that MAGE-A proteins can switch off the association between p53 and its responsive genes by inhibiting p53-dependent transcription. For example, the MAGE-A6 protein can inhibit expression of the p21 promoter. Low expression levels of MAGE-A results in the upregulation of genes, including p21, MDM2 and PUMA (29). As the present study found MAGE-A6 was downregulated by transfection of ROP16, it was hypothesized that, in SH-SY5Y cells, the downregulated expression of MAGE-A6 contributed to the expression of $\mathrm{p} 21$, therefore, $\mathrm{p} 53$-dependent apoptosis may have been partly stimulated.

The typical characteristic of ROP16 is that it can rapidly migrate into the nucleus of host cells following the invasion of T. gondii. It is essential to investigate the effect of ROP16 on gene transcription as the nucleus has the ability to control vital activities of the cell through the regulation of gene transcription and translation. Several DEGs were found to be involved in transcriptional regulation. The homeobox (HOX) genes encode conserved transcription regulators and govern processes, including morphogenesis and cell differentiation in vertebrate embryonic development and development of the central nervous system, where their expression is restricted and complex. It has been reported that, in human adipose tissue, the HOX gene network regulates the transcription of adipogenesis (30). In the present study, it was found that the majority of genes were upregulated, with the exception of HOXC11, which was downregulated. As HOX genes were differentially expressed due to the transfection of ROP16 in the present study, it was hypothesized that HOX genes may function critically in SH-SY5Y. However, the mechanism underlying the transcriptional regulation of HOX genes and their individual functions requires further investigation. The variation in gene expression in host cells under different physiological condition has been a key focus of investigations. The use of microarray technology assists in detecting alterations at a genome-wide level, which provides substantial benefits for experiments. In the process of altering gene expression in host cells, it is clear that pathogen proteins located in the nucleus are important. Thus, the examination of ROP16, a parasitic protein injected into the nucleus, contributed to improving comprehension of the pathogenic mechanisms of Toxoplasma.

In conclusion, the present study suggested that ROP16 transfection caused host cell alterations in the expression of multiple genes. These genes, particularly those involved in the processes of nervous system development, apoptosis and transcriptional regulation, may be vital in the response of host cells against $T$. gondii infection, and contribute to the pathogenesis of Toxoplasma. Novel candidate genes obtained through microarray analysis may provide insight for further investigations of its pathogenesis and therapeutic methods.

\section{References}

1. John B, Ricart B, Tait Wojno ED, Harris TH, Randall LM, Christian DA, Gregg B, De Almeida DM, Weninger W, Hammer DA and Hunter CA: Analysis of behavior and trafficking of dendritic cells within the brain during toxoplasmic encephalitis. PLoS Pathog 7: e1002246, 2011.

2. Montoya JG and Remington JS: Management of Toxoplasma gondii infection during pregnancy. Clin Infect Dis 47: 554-566, 2008

3. Tanaka S, Nishimura M, Ihara F, Yamagishi J, Suzuki Y and Nishikawa Y: Transcriptome analysis of mouse brain infected with Toxoplasma gondii. Infect Immun 81: 3609-3619, 2013.

4. Boothroyd JC: Have it your way: How polymorphic, injected kinases and pseudokinases enable Toxoplasma to subvert host defenses. PLoS Pathog 9: e1003296, 2013.

5. Dubremetz JF: Rhoptries are major players in Toxoplasma gondii invasion and host cell interaction. Cellular Microbiol 9: 841-848, 2007.

6. Mueller C, Klages N, Jacot D, Santos JM, Cabrera A, Gilberger TW, Dubremetz JF and Soldati-Favre D: The Toxoplasma protein ARO mediates the apical positioning of rhoptry organelles, a prerequisite for host cell invasion. Cell Host Microbe 13: 289-301, 2013.

7. Taylor S, Barragan A, Su C, Fux B, Fentress SJ, Tang K, Beatty WL, Hajj HE, Jerome M, Behnke MS, et al: A secreted serine-threonine kinase determines virulence in the eukaryotic pathogen Toxoplasma gondii. Science 314: 1776-1780, 2006.

8. Boothroyd JC and Dubremetz JF: Kiss and spit: The dual roles of Toxoplasma rhoptries. Nat Rev Microbiol 6: 79-88, 2008.

9. Saeij JP, Coller S, Boyle JP, Jerome ME, White MW and Boothroyd JC: Toxoplasma co-opts host gene expression by injection of a polymorphic kinase homologue. Nature 445: 324-327, 2007.

10. Jensen KD, Hu K, Whitmarsh RJ, Hassan MA, Julien L, Lu D, Chen L, Hunter CA and Saeij JP: Toxoplasma gondii rhoptry 16 kinase promotes host resistance to oral infection and intestinal inflammation only in the context of the dense granule protein GRA15. Infect Immun 81: 2156-2167, 2013.

11. Mi H, Muruganujan A and Thomas PD: PANTHER in 2013: Modeling the evolution of gene function, and other gene attributes, in the context of phylogenetic trees. Nucleic Acids Res 41 (Database Issue): D377-D386, 2013.

12. Mi H, Muruganujan A, Casagrande JT and Thomas PD: Large-scale gene function analysis with the PANTHER classification system. Nature Protoc 8: 1551-1566, 2013.

13. Chen J, Bardes EE, Aronow BJ and Jegga AG: ToppGene suite for gene list enrichment analysis and candidate gene prioritization. Nucleic Acids Res (Web Server Issue) 37: W305-W311, 2009.

14. Szklarczyk D, Franceschini A, Wyder S, Forslund K, Heller D, Huerta-Cepas J, Simonovic M, Roth A, Santos A, Tsafou KP, et al: STRING v10: Protein-protein interaction networks, integrated over the tree of life. Nucleic Acids Res 43 (Database Issue): D447-D452, 2014.

15. Shannon P, Markiel A, Ozier O, Baliga NS, Wang JT, Ramage D, Amin N, Schwikowski B and Ideker T: Cytoscape: A software environment for integrated models of biomolecular interaction networks. Genome Res 13: 2498-2504, 2003.

16. Bader GD and Hogue CW: An automated method for finding molecular complexes in large protein interaction networks. BMC Bioinformatics 4: 2, 2003 
17. Livak KJ and Schmittgen TD: Analysis of relative gene expression data using real-time quantitative PCR and the 2(-Delta Delta C(T)) Method. Methods 25: 402-408, 2001

18. Chen J, Xu H, Aronow BJ and Jegga AG: Improved human disease candidate gene prioritization using mouse phenotype. BMC Bioinformatics 8: 392, 2007.

19. Dugas JC, Mandemakers W, Rogers M, Ibrahim A, Daneman R and Barres BA: A novel purification method for CNS projection neurons leads to the identification of brain vascular cells as a source of trophic support for corticospinal motor neurons. J Neurosci 28: 8294-8305, 2008.

20. Oh JW, Olman M and Benveniste EN: CXCL12-mediated induction of plasminogen activator inhibitor-1 expression in human CXCR4 positive astroglioma cells. Biol Pharm Bull 32: 573-577, 2009.

21. Carruthers VB and Suzuki Y: Effects of Toxoplasma gondii infection on the brain. Schizophr Bull 33: 745-751, 2007.

22. Mori K, Kanemura Y, Fujikawa H, Nakano A, Ikemoto H, Ozaki I, Matsumoto T, Tamura K, Yokota M and Arita N: Brain-specific angiogenesis inhibitor 1 (BAI1) is expressed in human cerebral neuronal cells. Neurosci Res 43: 69-74, 2002.

23. Zhu D, Li C, Swanson AM, Villalba RM, Guo J, Zhang Z, Matheny S, Murakami T, Stephenson JR, Daniel S, et al: BAI1 regulates spatial learning and synaptic plasticity in the hippocampus. J Clin Invest 125: 1497508, 2015.
24. Nagai T, Aruga J, Minowa O, Sugimoto T, Ohno Y, Noda T and Mikoshiba K: Zic2 regulates the kinetics of neurulation. Proc Natl Acad Sci USA 97: 1618-1623, 2000.

25. Tsend-Ayush E, O'Sullivan LA, Grützner FS, Onnebo SM, Lewis RS, Delbridge ML, Marshall Graves JA and Ward AC: RBMX gene is essential for brain development in zebrafish. Dev Dyn 234: 682-688, 2005.

26. Wang T, Zhou J, Gan X, Wang H, Ding X, Chen L, Wang Y, DU J, Shen J and Yu L: Toxoplasma gondii induce apoptosis of neural stem cells via endoplasmic reticulum stress pathway. Parasitology 141: 988-995, 2014.

27. Nyoman AD and Lüder CG: Apoptosis-like cell death pathways in the unicellular parasite Toxoplasma gondii following treatment with apoptosis inducers and chemotherapeutic agents: A proof-of-concept study. Apoptosis 18: 664-680, 2013.

28. Iwasa H, Kudo T, Maimaiti S, Ikeda M, Maruyama J, Nakagawa K and Hata Y: The RASSF6 tumor suppressor protein regulates apoptosis and the cell cycle via MDM2 protein and p53 protein. J Biol Chem 288: 30320-30329, 2013.

29. Marcar L, MacLaine NJ, Hupp TR and Meek DW: Mage-A cancer/testis antigens inhibit p53 function by blocking its interaction with chromatin. Cancer Res 70: 10362-10370, 2010.

30. Cantile M, Procino A, D'armiento M, Cindolo L and Cillo C: HOX gene network is involved in the transcriptional regulation of in vivo human adipogenesis. J Cell Physiol 194: 225-236, 2003. 